\title{
Miscibility-Dispersion, Interfacial Strength and Nanoclay Mobility Relationships in Polymer Nanocomposites
}

\author{
Javier Carretero-González*, ${ }^{\text {,a, }}$, Haris Retsos ${ }^{\mathrm{b}}$, Emmanuel P. Giannelis ${ }^{\mathrm{b}}$, Tiberio A. Ezquerrac, Marianella \\ Hernández ${ }^{\mathrm{a}}$ and Miguel A. López-Manchado*,a \\ ${ }^{a}$ Instituto de Ciencia y Tecnología de Polimeros, CSIC, Madrid 28006, Spain \\ E-mail: jcarretero@ictp.csic.es and lmanchado@ictp.csic.es \\ ${ }^{b}$ Department of Material Science and Engineering, Cornell University, Ithaca, New York 14853 \\ ${ }^{c}$ Instituto de Estructura de la Materia, CSIC, Madrid 28006, Spain
}

\section{${ }_{10}$ Received (in $\left.X X X, X X X\right)$ Xth $X X X X X X X X X 200 X$, Accepted Xth $X X X X X X X X X 200 X$ First published on the web Xth $X X X X X X X X X 200 X$ DOI: $10.1039 / b 000000 x$}

Fully dispersed layered silicate nanoparticles (nanoclay) in a polymer matrix have provided a new class of multi-functional materials exhibiting several performance improvements over 15 conventional composites. Yet challenges with miscibility and interfacial strength might prevent nanocomposites from realizing their full potential. In this paper we demonstrate the effect of the chemical characteristics of the nanoclay on the miscibility and dispersion in the polymer matrix as well as on the interfacial strength of the bound polymer and the nanoclay mobility, all of which determine the macroscopic properties of the nanocomposite.

\section{Introduction.}

The effect of nanoscopic platelets of clay in the morphology of homopolymers, ${ }^{1}$ block-copolymers ${ }^{2}$ and polymer blends, ${ }^{3}$ as well as on their dynamic processes ${ }^{4-6}$ have 25 been commonly attributed to the high aspect ratio of the rigid nanoclay layers and to the large interfacial contact area between the clay and the polymer matrix. ${ }^{7}$ These effects have a profound impact on the mechanical properties of the nanocomposites due to nanoscale reinforcement. Despite these 30 promising results, and continous efforts by researchers worldwide to control the dispersion of nanoclay in the polymer, other aspects related to controlling the interfacial strength between the clay and the polymer and, thus, the properties of the nanocomposites remain a challenge.

35

Theoretical $^{8}$ and experimental ${ }^{9}$ studies provide evidence that polymer and nanoparticle mobility prevents the failure of polymer nanocomposites during deformation, by introducing an additional dissipative energy mechanism through the 40 formation of temporary cross-links between the polymer chains and the inorganic surface of the nanofiller. Although these studies conclude that the introduction of nanoparticles benefit the toughening and strengthening of polymeric matrices, the bound polymer also seems to play a key role in 45 the material's intrinsic properties. This is likely a consequence of the large surface area of nanoclay exposed to the polymer molecules, leading to a huge interfacial volume around the nanofillers. Zhou et al. ${ }^{10}$ recently concluded that this model of nanoparticle mobility will result in toughened 50 plastics only if both reduced interparticulate interactions and optima nanofiller/matrix interactions are guaranteed and accompanied by sufficient polymer mobility. Effective physical crosslinking thus requires high mobility and strong interaction between the nanoclay particles and the polymer, 55 allowing them to move together with the polymer chains during deformation.

In the present work we report the effect of the alkyl chain length, functionality and the grafting density of organic ${ }_{60}$ surfactant molecules, covering the surface of the nanoclay, on both the miscibility and the dispersion of nanoclay in natural rubber (NR) nanocomposites by means of $\mathrm{x}$-ray diffraction (XRD) and transmission electron microscopy (TEM). In an effort to understand the role of the interfacial region and the 65 mobility of the nanoclay in determining the properties of polymer nanocomposites, we have studied the polymer dynamics by means of broadband dielectric spectroscopy (BDS) and the microstructure under dynamic deformation by wide angle $x$-ray diffraction (WAXD). The anisotropic 70 organoclay nanoparticles are aligned within the rubber matrix by stretching. This allows us to monitor microstructural changes, including crystallization, by in situ WAXD. Both the alingnement capability and the interaction strength with the polymer matrix are ultimately determined by the chemical 75 characteristics of the nanoclay. These two aspects are crucial in designing new types of high-strength polymer nanocomposites.

Most studies on organoclay/polymer nanocomposites focus 80 on the effect that the extent of exfoliation has on the physical and mechanical properties of the material. However the improvement in polymer/NR matrices is dependent not only on the extent of exfoliation but also in other factors as the spatial distribution and orientation of the clay layers. A small 85 degree of aglomeration results in an inevitable decrease in performance. In the first part of this work, we study which 
chemical features of the nanoclay can improve the miscibility between the polymer and the nanoclay, and so permit nanoparticle dispersions on different length scales. In the second part of the paper the interplay between interfacial 5 strength and nanoparticle mobility in polymer nanocomposites is discussed.

\section{Results and discussion}

\section{${ }_{10}$ Sample processing}

A series of montmorillonite clays, organically modified with primary (single tails of varying lengths) and quaternary (stoichiometric and non-stoichiometric dimethyl 15 dehydrogenated tallow) alkyl ammonium salts, were mechanically mixed with NR, $\mathrm{M}_{\mathrm{w}} \sim 710 \mathrm{~K},\left(71010^{3} \mathrm{~g} \mathrm{~mol}^{-1}\right)$.

\section{Microstructure}

20 Table 1 describes the organoclays used as well as a comparison of the basal spacing, determined by XRD, before and after mixing with the polymer. As expected, a clear correlation between the number of carbon atoms in the primary alkyl chain (with similar surfactant concentration) or 25 the concentration of organic surfactant (for the same type of quaternary ammonium salt) and the initial basal distance for the organoclay is observed. However, after mixing and crosslinking of the rubber matrix the $d$-spacing is about the same in all the nanocomposites, thus suggesting that the final ${ }_{30} \mathrm{~d}$-spacing is independent of the initial organoclay spacing but it depends instead on the intercalated polymer.

Table 1. Selected data for the alkyl ammonium-modified layered silicates and their NR nanocomposites. Note that $\mathrm{C} X(X=12,14,16,18)$ and $\mathrm{Q}-Y$ $35(Y=\mathrm{I}$ and II $)$ are the samples based on primary and quaternary (ditallow) ${ }^{*}$ ammoniun alkyl chains, respectively.

\begin{tabular}{|c|c|c|c|c|}
\hline \multirow[b]{2}{*}{$\begin{array}{c}\text { Organocla } \\
y\end{array}$} & \multirow{2}{*}{$\begin{array}{c}\text { Organic } \\
\text { concentratio } \\
n \\
\text { (mmol/g } \\
\text { clay) }\end{array}$} & \multicolumn{2}{|c|}{$\mathrm{d}-$ Spacing $(\mathrm{nm})$} & \multirow[b]{2}{*}{$\begin{array}{l}\Delta \mathrm{d}-\text { Spacin } \\
\mathrm{g}(\mathrm{nm})\end{array}$} \\
\hline & & $\begin{array}{c}\text { Pure } \\
\text { organocla } \\
y\end{array}$ & $\begin{array}{c}\text { NR/organocla } \\
y\end{array}$ & \\
\hline $\mathrm{C} 12$ & $\sim 1.5$ & 1.7 & 3.8 & 2.1 \\
\hline $\mathrm{C} 14$ & $\sim 1.5$ & 1.8 & 3.8 & 2.0 \\
\hline $\mathrm{C} 16$ & $\sim 1.5$ & 2.0 & 3.7 & 1.7 \\
\hline $\mathrm{C} 18$ & $\sim 1.5$ & 2.1 & 3.7 & 1.6 \\
\hline Q-I & 1.2 & 2.9 & 3.8 & 0.9 \\
\hline Q-II & 0.9 & 2.4 & 4.1 & 1.7 \\
\hline
\end{tabular}

*The ditallow was a mixture of dimethylammonium surfactants with various carbon chain lengths of $c a .65 \%$ of $\mathrm{C} 18,30 \%$ of $\mathrm{C} 16$, and $5 \%$ of

$\mathrm{C} 14$. The surfactant concentration ( $\mathrm{mmol} \mathrm{g}^{-1}$ clay) was determined by

40 thermogravimetric analysis after Soxhlet extraction of the organomodified clay particles.
Nevertheless, as seen in Figure 1, sharper and better defined X-ray diffraction peaks $((001)$ reflections $)$ are obtained for the nanocomposites containing the layered 50 silicate nanoparticles modified with quaternary ammonium salt, Q-I and Q-II (Fig. 1, right). This means that more order is retained between the nanoclay layers after polymer mixing in the presence of quaternary ammonium salts. Differences in miscibility as a consequence of the differences in chemical 55 surface modification of the nanoclay is also clearly evident in the TEM analysis shown in Figures 2 and 3.
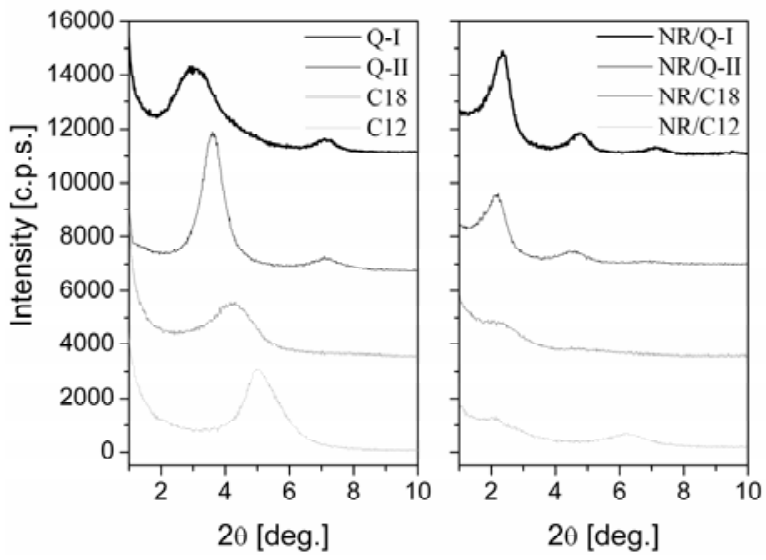

60

Figure 1. Left: XRD plots of Q-I (dark line), Q-II (thin line), C18 (medium line) and C12 (light line) nanoclays. Right: XRD plots of NR/Q-I (dark line), NR/Q-II (thin line), NR/C18 (medium line) and NR/C12 (light line) nanocomposites.

65

TEM images of the nanocomposite system containing the nanoclay organomodified with the linear alkyl ammoniun salt $70(\mathrm{C} 18)$ are shown in Figure 2. As observed in Figure 2a, the silicate is present as both individual layers, as well as small multilayer stacks (tactoids). Focusing in more detail (Figure $2 b, 2 c$ and $2 d$ ) on the differents areas of the sample, we find that large aggregates co-exist with the individual and smaller 75 tactoids mentioned above. Moreover, besides the breaking apart of big silicate particles into smaller clay particles, a peeling effect from the aggregates forming individual and isolated nanoclay particles, as a consequence of shearing during mechanical mixing, was also observed for the layered 80 silicates organomodified with the linear surfactant (Figure 2c). 

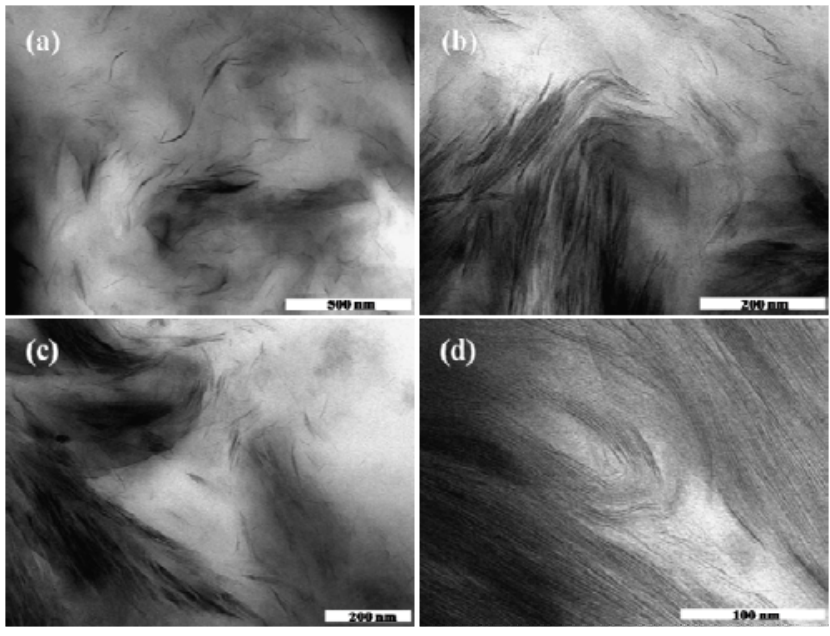

(d)

Figure 2. Different magnifications $(20 \mathrm{~K} \times-50 \mathrm{~K} \times)$ TEM images for NR/C18 nanocomposite.

5 In contrast, the use of organoclays functionalized with a quaternary ammonium salt reduces the micron size agglomerates to smaller tactoids instead of peeling them into individual platelets, as shown in Figure 3. A highly homogeneous NR nanocomposite system was obtained 10 regardless of a stoichiometric Q-I (Figure 3Ia and 3IIa) or non-stoichiometric Q-II (Figure 3Ib and 3IIb) amount of ammoniun present. The nanocomposites with Q-type nanoclays exhibit a microstructure characterized by nanoclay particles arranged in small groups of finely dispersed clay 15 tactoids (containing $\sim 10$ layers). These tactoids are largely isolated and separated by distances of $\sim 10-50 \mathrm{~nm}$ instead of forming a rigid filler network. These observations suggest a different intercalation/exfoliation mechanism for the two types of organic surfactants, each of them exhibiting a 20 different level of miscibility with the polymer matrix.
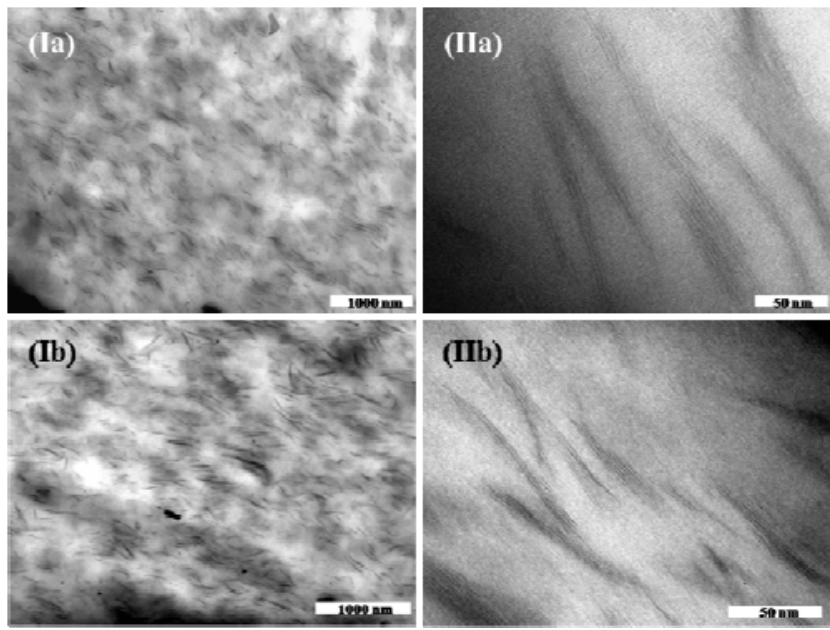

(III)

Figure 3. Low (I) and high (II) magnification TEM images for (a) NR/Q-I and (b) NR/Q-II nanocomposites filled with $15 \mathrm{phr}$ of nanoclay.

25 Due to its homogeneity, the microstructures shown in Figure 3 may represent an ideal system to study the interplay between the interfacial strength and the mobility of the nanoclay under stretching.

\section{${ }_{30}$ Dielectric Relaxation Spectroscopy}

Figure 4 shows the dielectric loss, $\varepsilon$ ", versus temperature for pristine vulcanized NR and for the nanocomposite containing Q-I nanoclay (15 phr). It is well known that cis35 polyisoprene (a major component in NR) exhibits two relaxations. ${ }^{11}$ One of them is related to the segmental motion (segmental mode) and a slower one related to the relaxation of whole chains (normal mode) ${ }^{12}$. The normal mode disappears after croslinking due to supression of large-scale motions of 40 the dipole oriented parallel to the polymer backbone. In our case, vulcanized NR exhibits an intense relaxation around $237.5 \mathrm{~K}$, associated with the segmental mode (SM), accompanied at lower temperatures by another relaxation albeit of lower intensity. No significant changes in the 45 relaxation behavior were observed when the nanoclay was added. This indicates that the segmental motion of cis-polyisoprene is not disturbed by the addition of the nanoparticles. Consequently, both samples must have similar glass transition temperature values. For the NR 50 nanocomposite. a slower relaxation (SR) appears at higher temperatures $(\mathrm{T} \sim 325 \mathrm{~K})$. The strong increase of $\varepsilon$ ', at even higher temperatures can be associated to a Maxwell-Wagner-Sillars (MWS) process induced by the accumulation of charges at the polymer-nanoclay interfaces.

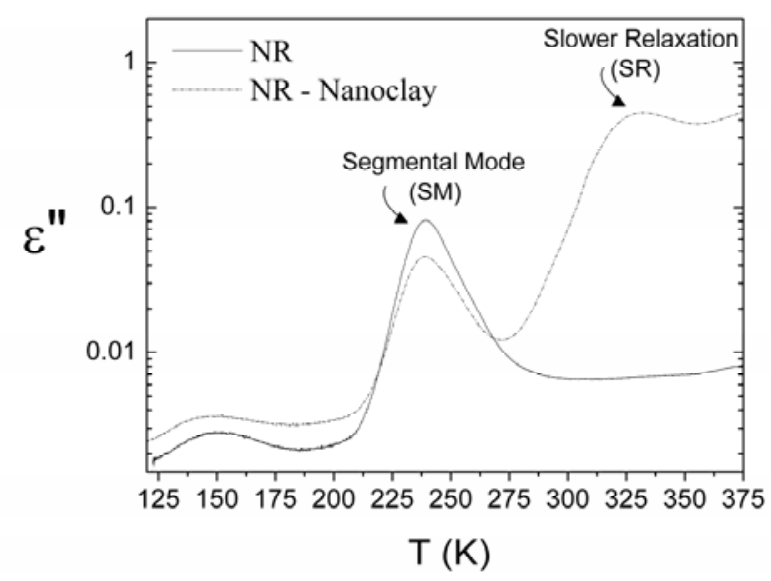

Figure 4. Temperature dependence of $\varepsilon "$ at $10^{3} \mathrm{~Hz}$ for the unfilled and nanocomposite NR samples after crosslinking. For vulcanized NR the main process is associated with the segmental mode (SM). For the 60 nanocomposite an additional slower relaxation (SR) appears at higher temperatures. 
The dielectric relaxations for NR and the nanocomposite as a fucntion of both temperature and frequency are shown in Fig. 5.
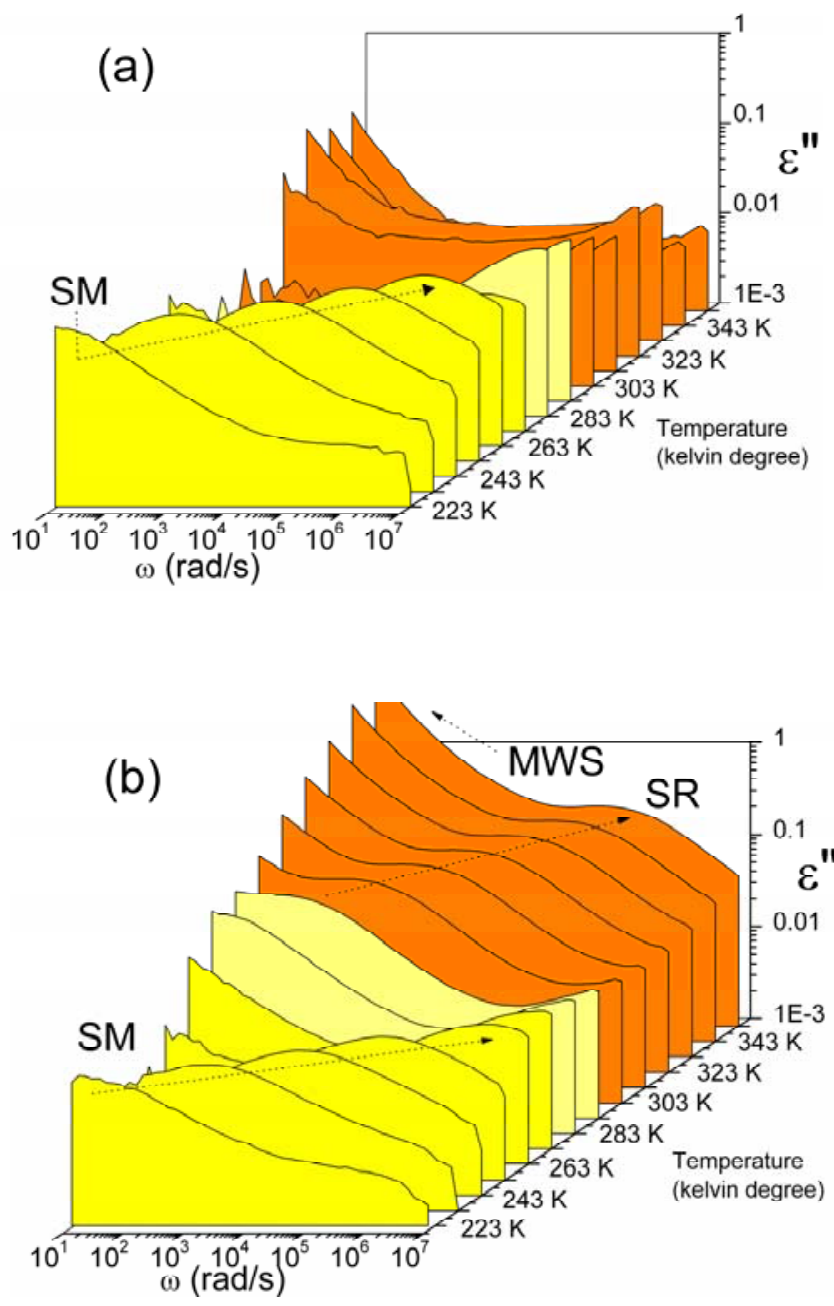

Figure 5. $\varepsilon$ " values for unfilled NR (a) and the NR nanocomposite containing $15 \mathrm{phr}$ of Q-I nanoclay (b) as a function of frequency and temperature.

The dielectric relaxation spectra were analyzed using the Havriliak-Negami formulation. From this analysis the 15 relaxation time of the different processes were extracted and they are represented in Fig. 6 as a function of the reciprocal temperature. As expected, the relaxation times for the segmental mode of both vulcanized NR and the NR/Q-I nanocomposite follows the characteristic ${ }_{20}$ Volgel-Fulcher-Tamann (VFT) dependence

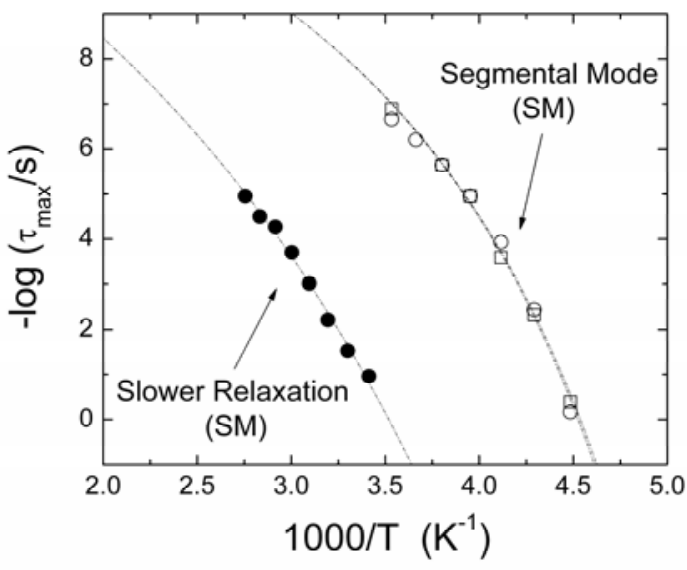

Figure 6. Dependence of the relaxation time corresponding to the maximum loss $\left(\tau_{\max }\right)$ with the reciprocal temperature for the segmental

30 relaxation (open symbols) for the unfilled polymer matrix ( $\square$ ) and the nanocomposite $(\circ)$. The slower relaxation mode assigned to the interfacial adsorbed polymer in the nanocomposites is also plotted $(\bullet)$. Dashed lines represent Volger-Fulcher-Tamann fits for each relaxation.

35 Table 2. $\mathrm{T}_{\mathrm{g}}$ and VFT parameters for the unfilled sample and the NR-Q-I nanocomposite.

\begin{tabular}{cccc} 
Sample & $\mathrm{B}$ & $\mathrm{T}_{0}(\mathrm{~K})$ & $\mathrm{T}_{\mathrm{g}}(\mathrm{K})$ \\
NR(SM) & 868 & 159.0 & 232 \\
NR15 Q-I (SM) & 873 & 158.6 & 233 \\
NR 15 Q-I (SR) & 1976 & 142.9 & 325 \\
\hline
\end{tabular}

The slower relaxation (SR) of the NR/Q-I nanocomposite also seems to follow a VFT behaviour with the parameters 40 presented in Table 2. We can hypothesise that the SR appears as a consequence of the interaction of the rubber chains with the nanoclay surfaces inducing a slower segmental mode with a new effective $\mathrm{T}_{\mathrm{g}}$ of $\sim 90 \mathrm{~K}$ higher than in the neat polymer (i.e. in the absence of the nanoclay).

45

\section{Strain Induced Crystallization}

The alignment of the nanoclay in the polymer matrix upon 50 stretching was evaluated by in-situ monitoring the evolution of the integrated intensity of the $I_{200}$ reflection of the crystalline phase of NR. Figure $7(\mathrm{Up})$ shows the dependence of $\mathrm{I}_{200}$ with the deformation ratio $\alpha$ during stretching. The corresponding stress-strain curve for both the unfilled NR and 55 the corresponding nanocomposites are shown in Figure 7.

$$
F_{\text {max }}=F_{0} \exp \left[-D T_{0} /\left(T-T_{0}\right)\right] .
$$



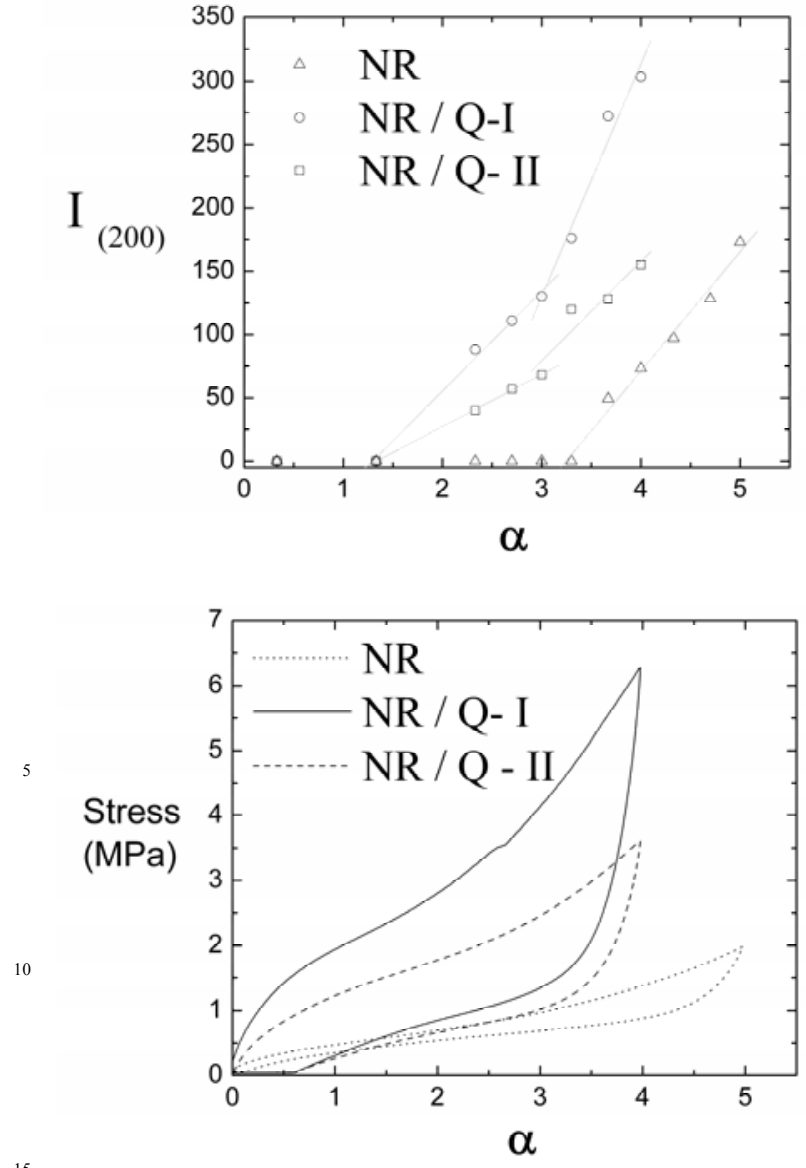

15

Figure 7. Up: Variation of the integrated intensity of the 200 reflection during the stretching process for NR/Q-I ( $\circ$ ), NR-QII ( $\square$ ) and unfilled NR $(\Delta)$. Down: stress-strain curves for the NR/Q-I (solid line), NR/Q-II 20 (dashed line) and unfilled NR (dotted line) samples.

Crystallization of neat NR seems to occur in a single step once a critical value of strain $\alpha=3.4$ is reached. In contrast crystallization in the nanocomposites commences at $\alpha \approx 1.2$ 25 and appears to follow a more complex, two-step pattern. ${ }^{13}$ Although the $I_{200}$ intensity values are directly related to the amount of NR crystallites formed during stretching,${ }^{14}$ we must take into account that the motion of the nanoclay particles and the motion of the polymer chains must be coupled. The first 30 step for $\alpha$-values $<3$ is most likely related to the orientation and alignment of the highly anisotropic nanoclay particles. In a second step $(3 \leq \alpha \leq 4)$, these highly anisotropic nanoparticles can be completely aligned along the direction of the deformation and thus a physical network is formed. This ${ }_{35}$ physical network may favor the alignment of the rubber chains and the crystallization rate could increase. Moreover, the excess of loosely bound surfactant seems to facilitate the strain-induced crystallization process in NR/Q-I in comparison with NR/Q-II. The change in the crystallizability 40 of the polymer as well as the mobility of the nanoclay can be attributed to the presence of surfactant molecules surrounding the nanoclay fillers. This effect is not expected to be due to the optimum thermodinamic interaction ${ }^{15}$ between the organic alkyl chains of surfactant and the polymer at the interface, but 45 rather to the lower friction of polymer chains with the inorganic surface as a consequence of the higher amount of inorganic surface covered by the surfactant alkyl-chains. ${ }^{16}$ This is likely also directly related to an increase in dispersion quality as well as a decrease in processing time as a result of ${ }_{50}$ employing a quaternary branched alkyl chain instead of a linear ammoniun salt as previously demonstrated. Such a change would certainly increase the "self-lubricating" effect among the grafted nanoparticles and the polymer. As a result, the final crystalline content of the nanocomposites is higher $5 s$ and there is a more effective toughenning as shown in Figure 7 down.

\section{Conclusions.}

${ }_{60}$ The influence of the chemical characteristics of the nanoclay on the microstructure and the dynamics of nanoclay-natural rubber nanocomposites has been discussed. We have shown by using broadband dielectric spectroscopy that the nanoclaypolymer interaction induces a new relaxation process which is ${ }_{65}$ slower than the segmental relaxation of the rubber matrix. In addition, in-situ synchrotron $\mathrm{x}$-ray experiments give an indirect measurement of the mobility of the nanoclay during uniaxial elongation. Our results emphasize the importance of the chemical surface modification of nanoclay as being crucial 70 to promote an optimum mechanical reinforcement.

\section{Experimental}

Synthesis of the Nanocomposites: The natural rubber sample 75 was kindly supplied by Malaysian Rubber (Berhad, Malaysia) under the trade name NR CV60 (mooney viscosity ML (1+4) at $100{ }^{\circ} \mathrm{C}=60$ ). Nanoclay Q-I (non-stoichiometric dimethyl dehydrogenated tallow ion-exchanged montmorillonite) was supplied by Southern Clay Products (Gonzales, TX, USA) and ${ }_{80}$ Q-II was prepared from Q-I after Soxhlet extraction of Q-I. nanoclays based on linear ammonium alkyl chains surfactants (C12-C18) were prepared by ion exchange with sodium montmorillonite (Southern Clay Products) and the organic surfactants (Aldrich) at $343 \mathrm{~K}$. The formulation of natural 85 rubber compounds expressed in phr (parts per hundred rubber) is as follows: sulfur (2.5), $\mathrm{ZnO}$ (5), stearic acid (1), MBTS (1), antioxidant PBN (1) and nanoclays (variable). Nanocomposites were prepared in an open two-roll laboratory mixing mill at room temperature. Vulcanization was carried 90 out in an electrically heated hydraulic press, at $150{ }^{\circ} \mathrm{C}$ at the optimum cure time $\left(t_{90}\right)$ previously determined with a rubber process analyzer (RPA Alpha Technologies).

$X$-Ray diffraction: $\mathrm{X}$-Ray diffraction was performed in a $\theta-\theta$ diffractometer with an integrated germanium detector, using a ${ }_{95} \mathrm{CuK} \alpha$ source with a wavelength of $1.54 \AA$ and a scan rate of $2^{\circ} \min ^{-1}$.

Microscopy: The dispersion of the nanoparticles in the rubber matrix was determined through transmission electron microscopy (TEM), using a Technai T12 TEM operated at an 100 accelerating voltage of $120 \mathrm{kV}$. TEM samples $(\sim 40 \mathrm{~nm})$ were 
prepared by sectioning at $-160^{\circ} \mathrm{C}$ using a Leica Ultracut UCT ultracryomicrotome with a diamond knife.

Broadband Dielectric Spectroscopy: Dielectric spectroscopy measurements were performed using the Novocontrol Turnkey ${ }_{5}$ Concept N40 broadband spectrometer (Hundsangen, Germany). Sample discs were mounted in the dielectric cell (ZGS Alpha Active Sample Cell) between two parallel gold-plated electrodes (BDS 1301 model). The thickness of the gold-plated electrodes was $2 \mathrm{~mm}$ and the diameters were $1020 \mathrm{~mm}$ (upper) and $30 \mathrm{~mm}$ (down), respectively. The sample thickness was $1 \mathrm{~mm}$ and the diameter was about $15 \mathrm{~mm}$. The sample/electrode assembly was then mounted in the Novocontrol Quatro Cryosystem.

Synchrotron X-ray measurements: In situ stress-strain 15 experiments coupled with the synchrotron $\mathrm{X}$-ray measurements were carried out at the $\mathrm{X} 27 \mathrm{C}$ beamline in the National Synchrotron Light Source, Brookhaven National Laboratory. The wavelength was $0.1366 \mathrm{~nm}$. The deformation rate was $10 \mathrm{~mm} / \mathrm{min}$ and the experiments were carried out at 20 room temperature. The maximum strain value was $400 \%$ due to the slipping of the sample from the clamps. The two dimensional WAXD patterns were recorded using a MAR CCD camera. Exposure time for each image was $30 \mathrm{~s}$ and there was an interval of $5 \mathrm{~s}$ before the next exposure. The

25 diffraction angle was calibrated by $\mathrm{Al}_{2} \mathrm{O}_{3}$ standard (provided by the National Institute of Standards and Technology). The images were processed using "POLAR" software (Stony Brook Technology and Applied Research, Inc.). The crystallinity index (C.I.) was calculated from the integration 30 and correction of the equatorial 2D intensity WAXD profiles at the azimuthally range of \pm 75 using peak fit software.

\section{Acknowledgements}

35 The authors gratefully acknowledge the financial support of the Spanish Ministry of Science and Innovation (MICINN) through projects MAT 2004-00825 and MAT-2005-01768. JCG acknowledges support from MICINN in the form of a FPI grant and EPG acknowledges the support of 40 Award No. KUS-C1-018-02 made by King Abdullah University of Science and Technology (KAUST). Special thanks are due to Shigeyuki Toki and Benjamin S. Hsiao from Stony Brook University and the X27C beamline in the National Synchrotron Light Source at Brookhaven National Laboratory.

45

\section{References}

1 D. M. Lincoln, R. A. Vaia and R. Krishnamoorti, Macromolecules, 2004, 37, 4554.

502 A. S. Silva, C. A. Mitchell, M. F. Tse, H-C. Wang and R. Krishnamoorti, J. Chem. Phys., 2001, 115, 7166.

3 M. Arroyo, M. A. Lopez-Manchado, J. L. Valentin and J. Carretero, Comp. Sci. Tech., 2007, 67, 1330.

4 R. Krishnamoorti and E. P. Giannelis, Macromolecules, 55 1997, 30, 4097.

5 S. H. Anastasiadis, K. Karatasos, G. Vlachos, E. Manias and E. P. Giannelis, Phys. Rev. Lett., 2000, 84, 915.

6 S. Salinawal, S. K. Kumar and J. F. Douglas, Phys. Rev.

Lett., 2002, 89, 258301.
${ }_{60} 7$ R. A. Vaia, Mater. Today, 2004, 7, 32.

8 D. Gersappe, Phys. Rev. Lett., 2002, 89, 058301.

9 D. Shah, P. Maiti, D. D. Jiang, C. A. Batt and E. P. Giannelis, Adv. Mater. 2005, 17, 525.

10 H. Z. Tong, H. R. Wen, Z. R. Min, Q. Z. Min and L. M. ${ }_{65} \mathrm{Yu}$, Adv. Mater., 2007, 19, 2667.

11. W. H. Stockmayer and M. E. Baur, J. Am. Chem. Soc., 1964, 86, 3485.

12. K. Adachi and T. Kotaka, Macromolecules, 1984, 17, 120.

13. J. Carretero-González, H. Retsos, R. Verdejo, S. Toki, B. ${ }_{70}$ S. Hsiao, E. P. Giannelis, M. A. López-Manchado, Macromolecules, 2008, 41, 6763.

14. S. Toki, I. Sics, S. Ran, L. Liu, B. S. Hsiao, S. Murakami, K. Senoo and S. Kohjiya, Macromolecules 2002, 35, 6578.

15. R. A. Vaia and E. P. Giannelis, Macromolecules, 1997, 75 30, 7990.

16. E. Manias, H. Chen, R. Krishnamoorti, J. Genzer, E. J. Kramer and E. P. Giannelis, Macromolecules, 2000, 33, 7955. 